\title{
THE FORMATION OF GALAXY DISKS AND BULGES
}

\author{
P.R. WILLIAMS AND A.H. NELSON \\ Department of Physics and Astronomy, University of Wales \\ College Cardiff, PO Box 913, Cardiff, CF2 3YB
}

Using a TREESPH code modified to run on a parallel computer and a new algorithm for modeling star formation, we have investigated the formation of disk galaxies from idealized cosmological perturbations.

The initial conditions used were an isolated uniform density sphere in solid body rotation and in Hubble expansion. The total mass was $5 \times$ $10^{11} M_{\odot}, 10 \%$ gas and $90 \%$ dark matter. The star formation law used was a Schmidt law of index 1.5. Our simulations have shown that isolated initial conditions with no small scale noise can parent numerical galaxies which are very similar to those observed in the field. The resultant numerical galaxies were found to have four mass components: a dark matter halo, a thin gas disk, a stellar disk, and a spherical bulge. Also, the numerical galaxies were found to have the properties found in typical field spirals:

- Rotation curves were found to be flat over the galaxy disk and have a maximum velocity of $\sim 170 \mathrm{~km} \mathrm{~s}^{-1}$.

- The surface density profile was found to be well modeled with two exponential distributions, representing the disk and bulge components. The bulge and disk scale lengths were found to be typically $0.25 \mathrm{kpc}$ and $1.4 \mathrm{kpc}$ respectively.

- The star formation rate increased rapidly to $1000 M_{\odot} \mathrm{yr}^{-1}$ during collapse and decreased to $10 M_{\odot} \mathrm{yr}^{-1}$ within $1 \mathrm{Gyr}$.

- A spherical bulge component, supported by an isotropic velocity dispersion, formed during the collapse.

Steinmetz \& Müller (1995) have suggested that the size of the bulge component in a disk galaxy is determined by the initial level of small scale noise and the initial amount of random kinetic energy. Our simulations suggest that the properties of the bulge are more likely determined by the physical parameters of the initial perturbation.

\section{References}

Steinmetz M., \& Müller E. 1995, MNRAS, 276, 549 